Supporting Information

\title{
Triggering Amino Acid Detection by Atomistic Resolved \\ Tunneling Current Signals in Graphene Nanoribbon Devices for Peptide Sequencing
}

\author{
Tommaso Civitarese and Giuseppe Zollo* \\ Dipartimento di Scienze di Base e Applicate per l'Ingegneria, University of Rome "La \\ Sapienza", Via A. Scarpa 14-16, 00161 Rome, Italy \\ E-mail: giuseppe.zollo@uniroma1.it
}

Phone: +39 (0) 6 49766947. Fax: +39 (0) 644240183 

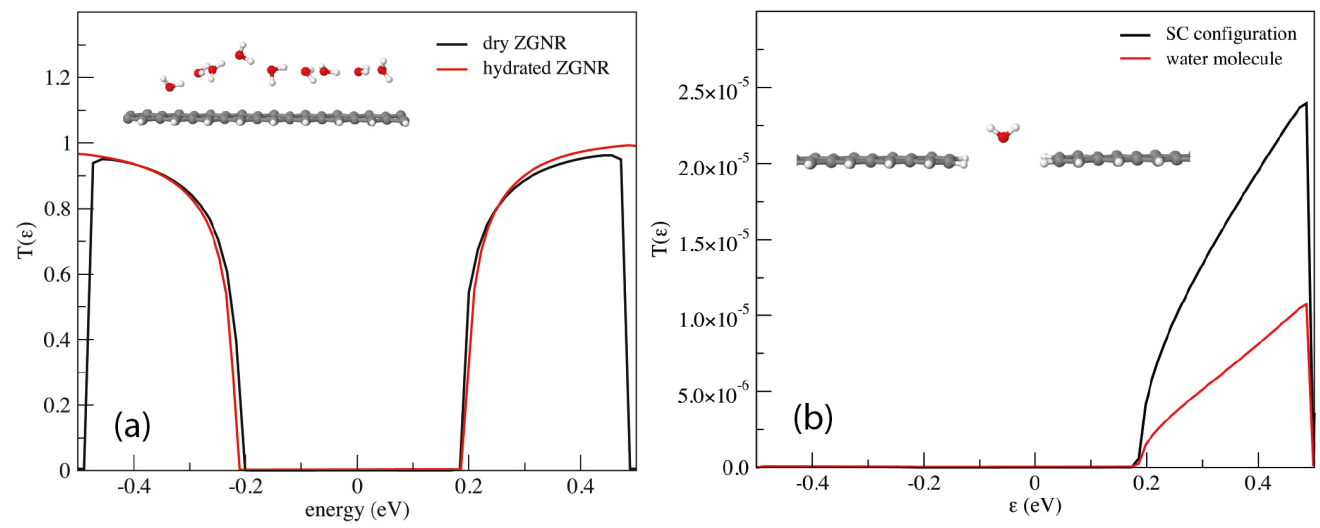

Figure S1: (a) Transmission function of the 2-ZGNR with waters adhering on the ribbon plane compared to the pristine one. The two transmission functions are very close thus showing that waters on the electrode plane do not affect the device conduction properties. (b) Transmission function of the 2-ZGNR nanogap with one water molecule between the leads. It is compared to the transmission function obtained with the side chain (SC) configuration of the Gly homo-peptide (the Gly side chain is in the middle of the gap) where the Gly signal ha a minimum. We can see that the tunneling current through the water molecule is one order of magnitude smller than the minimum current value measured for the Gly homo-peptide. In the insets are reported the corresponding analyzed configurations of water in the 2-ZGNR device.

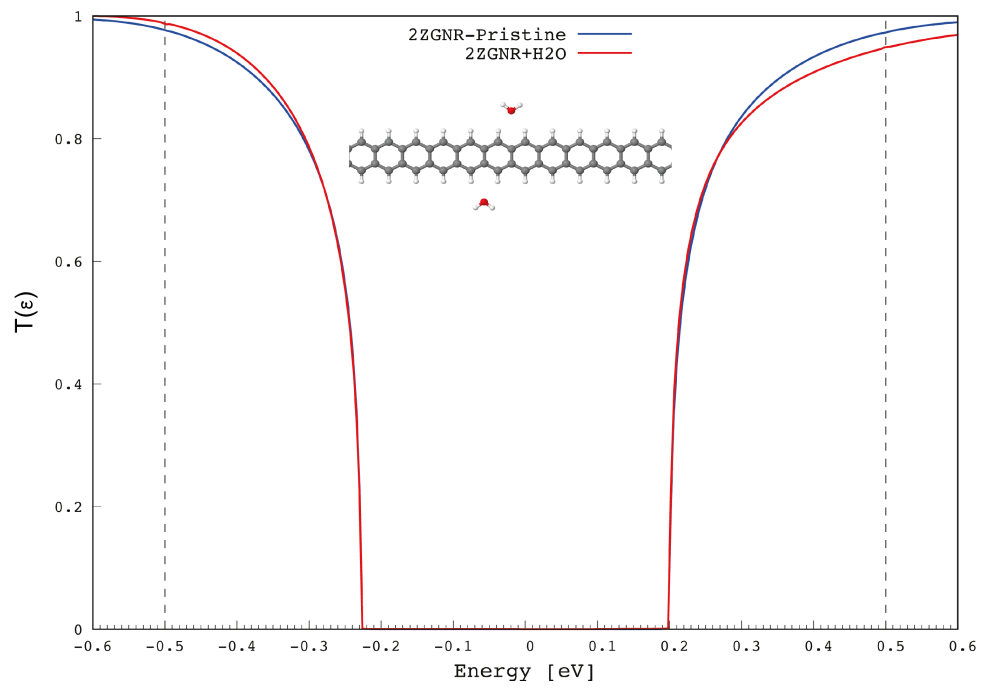

Figure S2: Transmission function of the 2-ZGNR with waters adhering on the ribbon edges as compared to the pristine one. Here the waters have been considered in the device region (see the inset). We can see that the transmission function obtained is very much close to the one of the pristine (without waters) nanogap. 


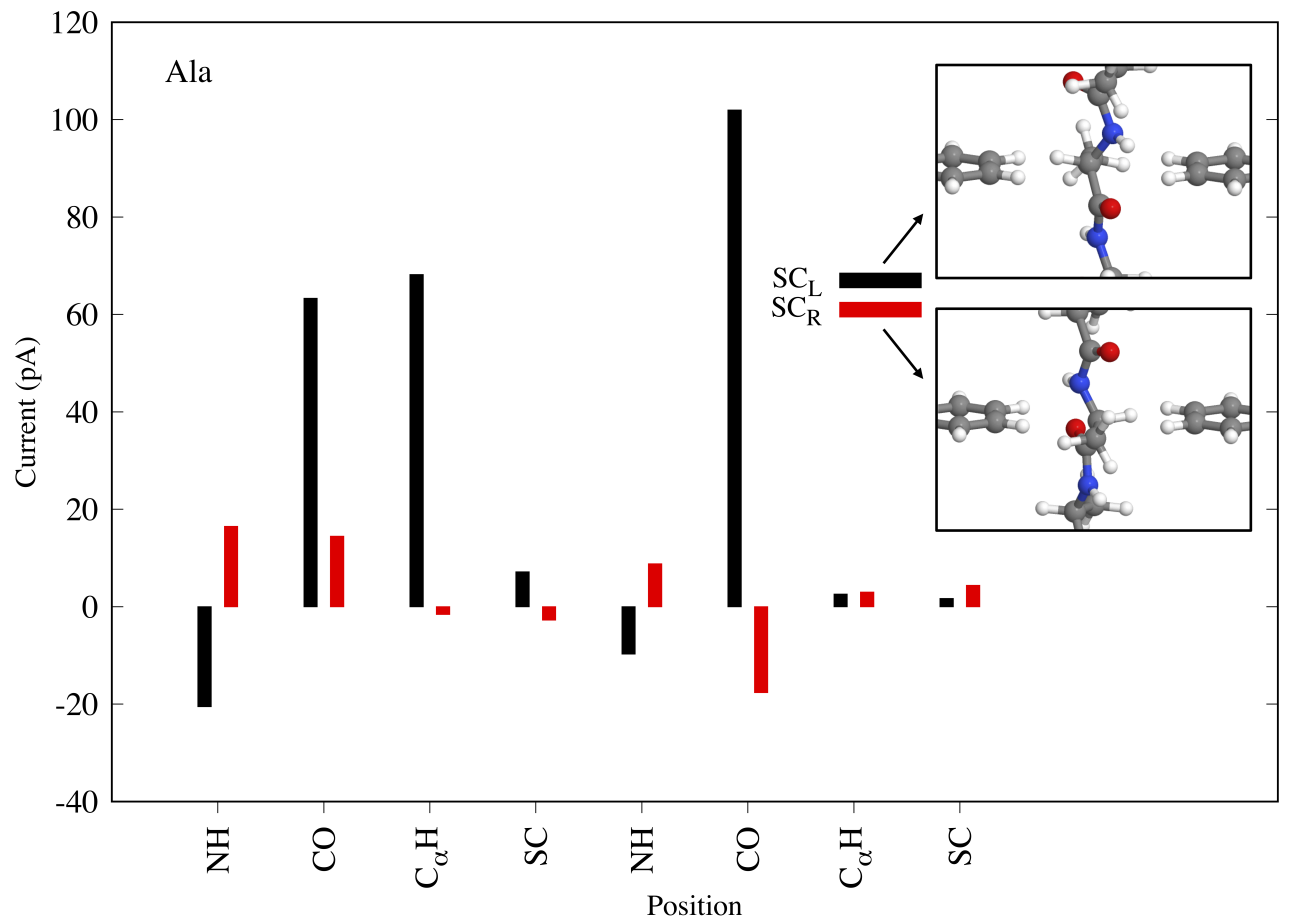

Figure S3: Bond currents injected from the left lead into the peptide for the SC configurations of Ala homopeptide with different orientation of the $C_{\alpha} \mathrm{H}$ bond either toward the left lead electron source $(\mathrm{L})$ or toward the right lead electron drain $(\mathrm{R})$. 


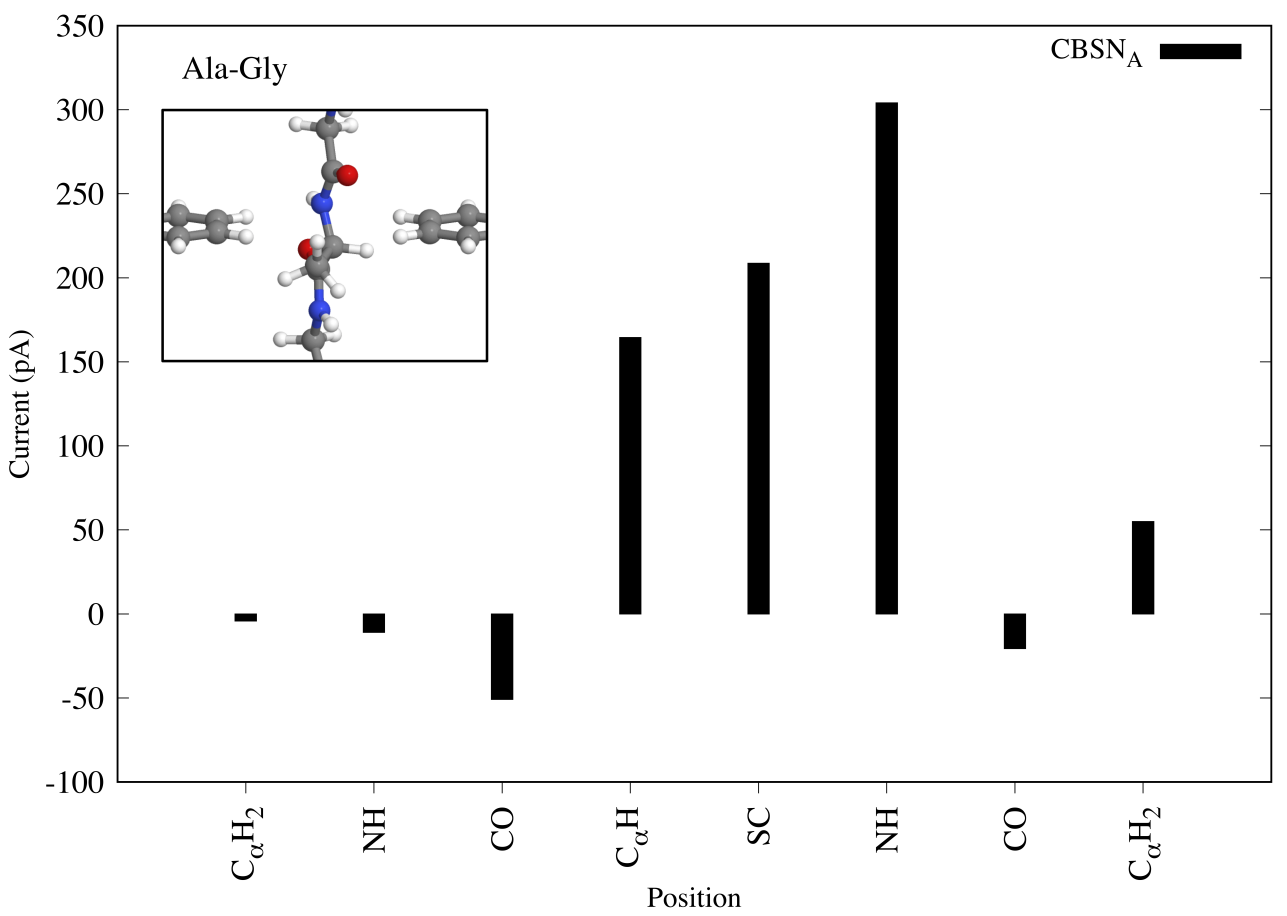

Figure S4: Bond currents injected from the left lead into the peptide for the $C B S N_{A}$ configurations of Ala-Gly heteropeptide. The label $C_{\alpha} H_{2}$ in the x-axis is referred to glycine amino acid while $C_{\alpha} \mathrm{H}$ is referred to alanine. 

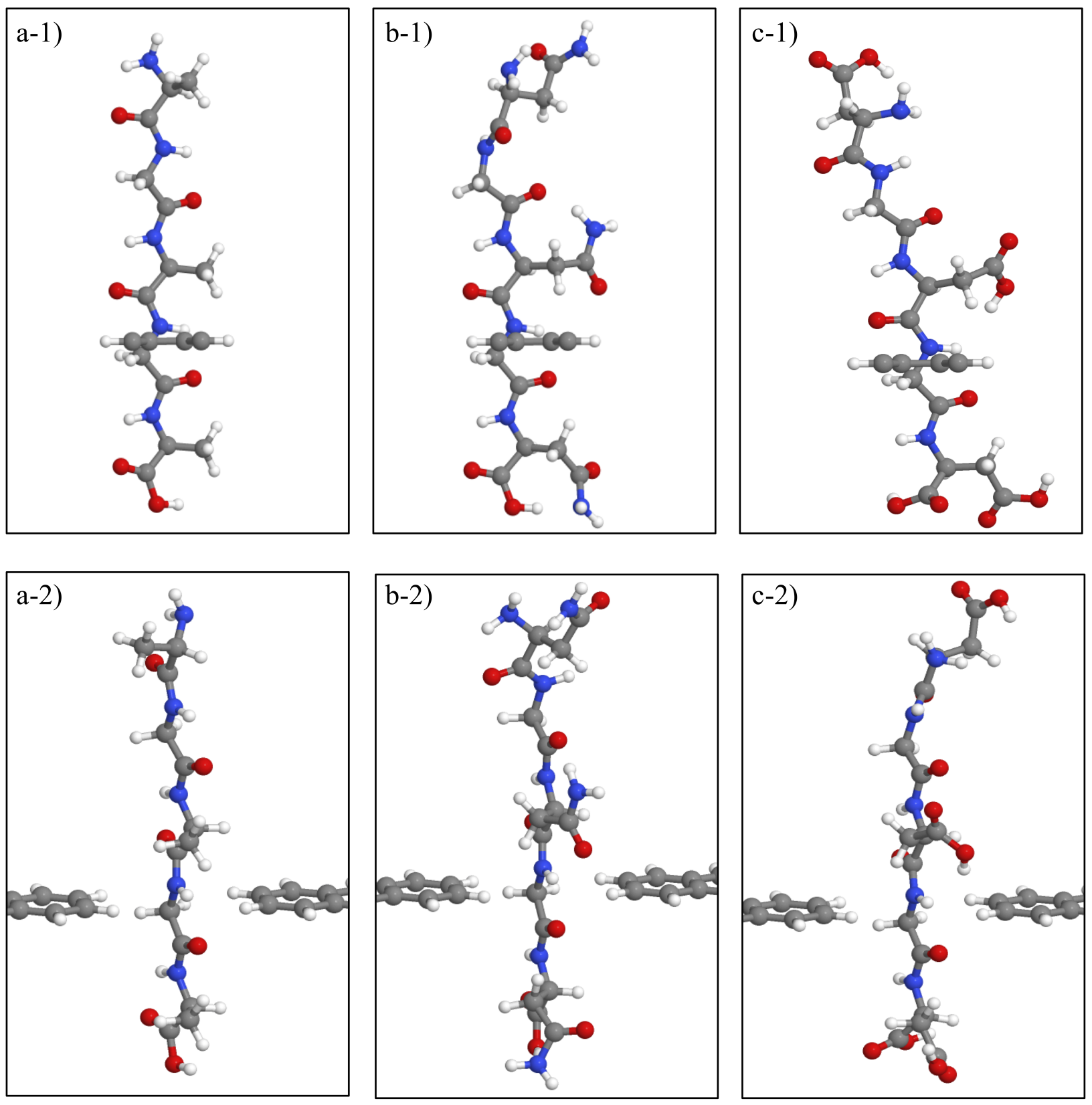

Figure S5: $C B S N_{G}$ configurations of heteropeptides Ala-Gly, Asn-Gly and Asp-Gly labelled respectively with the letter a-1, b-1, c-1 for the left lead side view and a-2, b-2 and c-2 for the perspective view. 


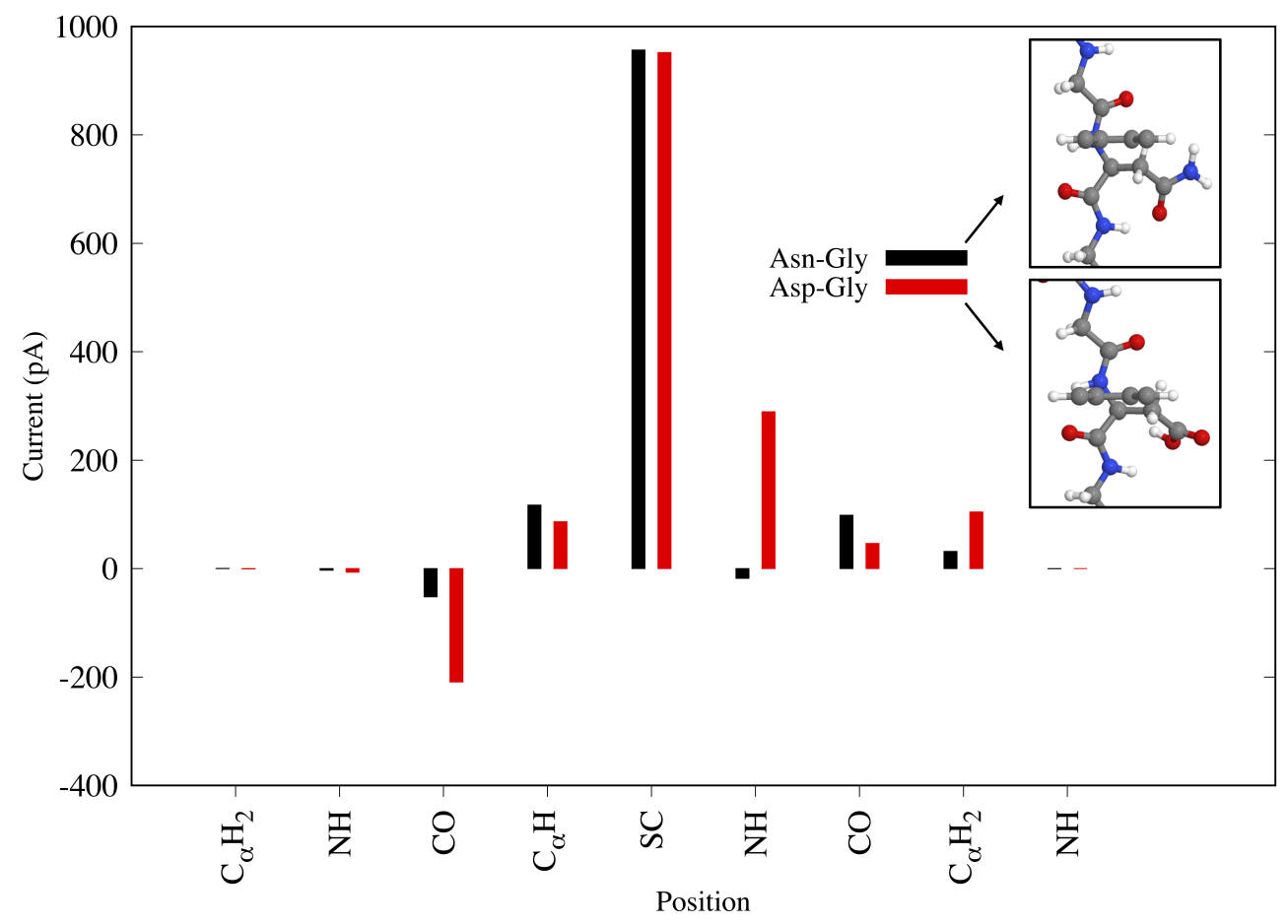

Figure S6: Bond currents injected from the left lead into the peptide for $N H 3_{N}$ and $C B S N_{D}$ configurations of, respectively, Asn-Gly and Asp-Gly heteropeptide. The two configurations represented are those corresponding to the main peaks in figure 7 in the article. In the boxes are reported the left lead side view of the two configurations analyzed. 


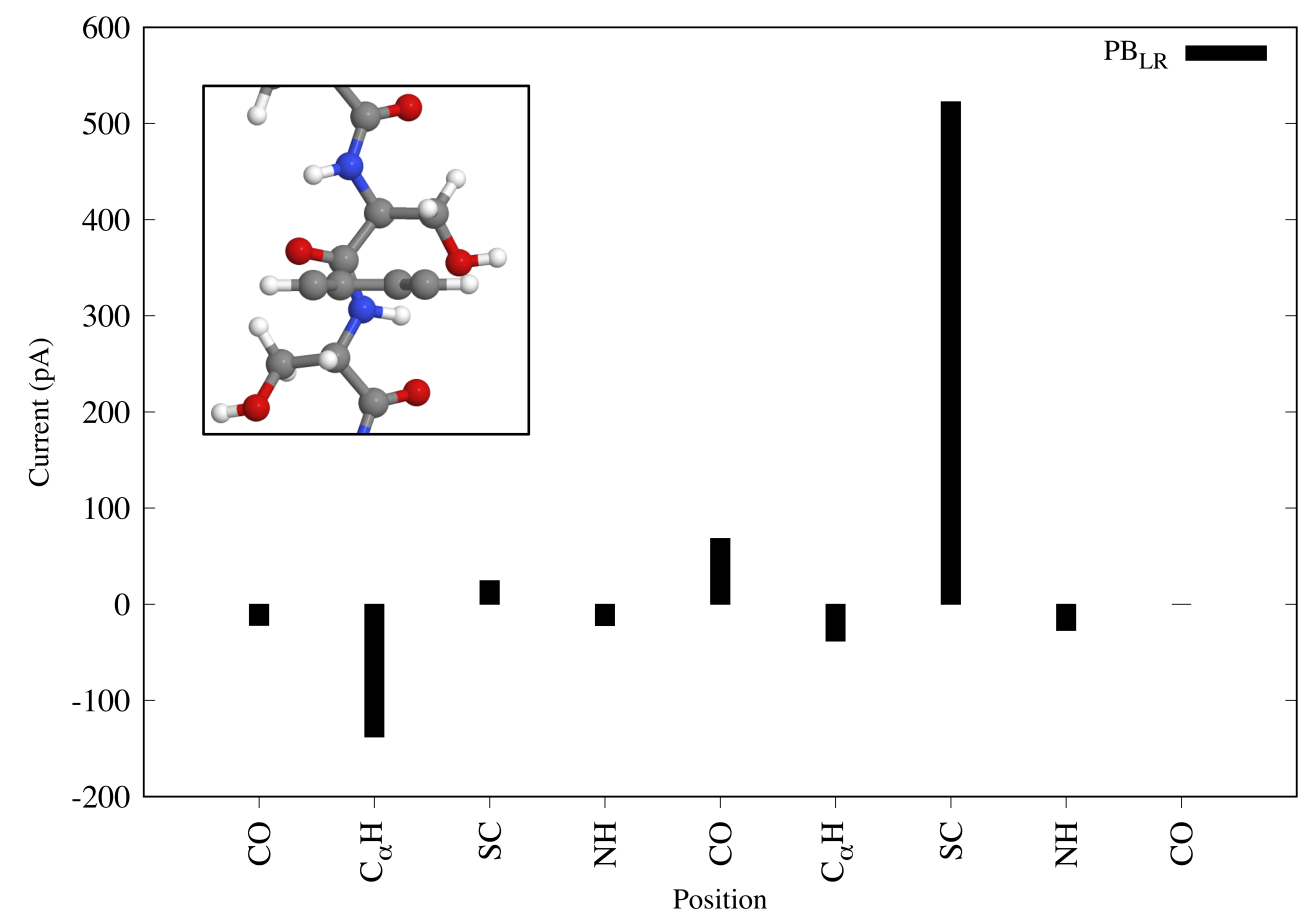

Figure S7: Bond currents injected from the left lead into the peptide for the $P B_{L R}$ configuration of Ser homopeptide. In the boxes is reported the left lead side view of the configuration analyzed. As it is possible to see, the main contribution to the peak at $P B_{L R}$ is due to the side chain (SC) located above the plane of the leads. If you decompose further the bond currents, dividing those that pass through the $C_{\beta}$ with the 2 hydrogens and those through the polar group $O H$, it emerges that only the $C_{\beta}$ contributes to the current reported with $534 \mathrm{pA}$; on the polar group it is possible to observe a weak reflection. 


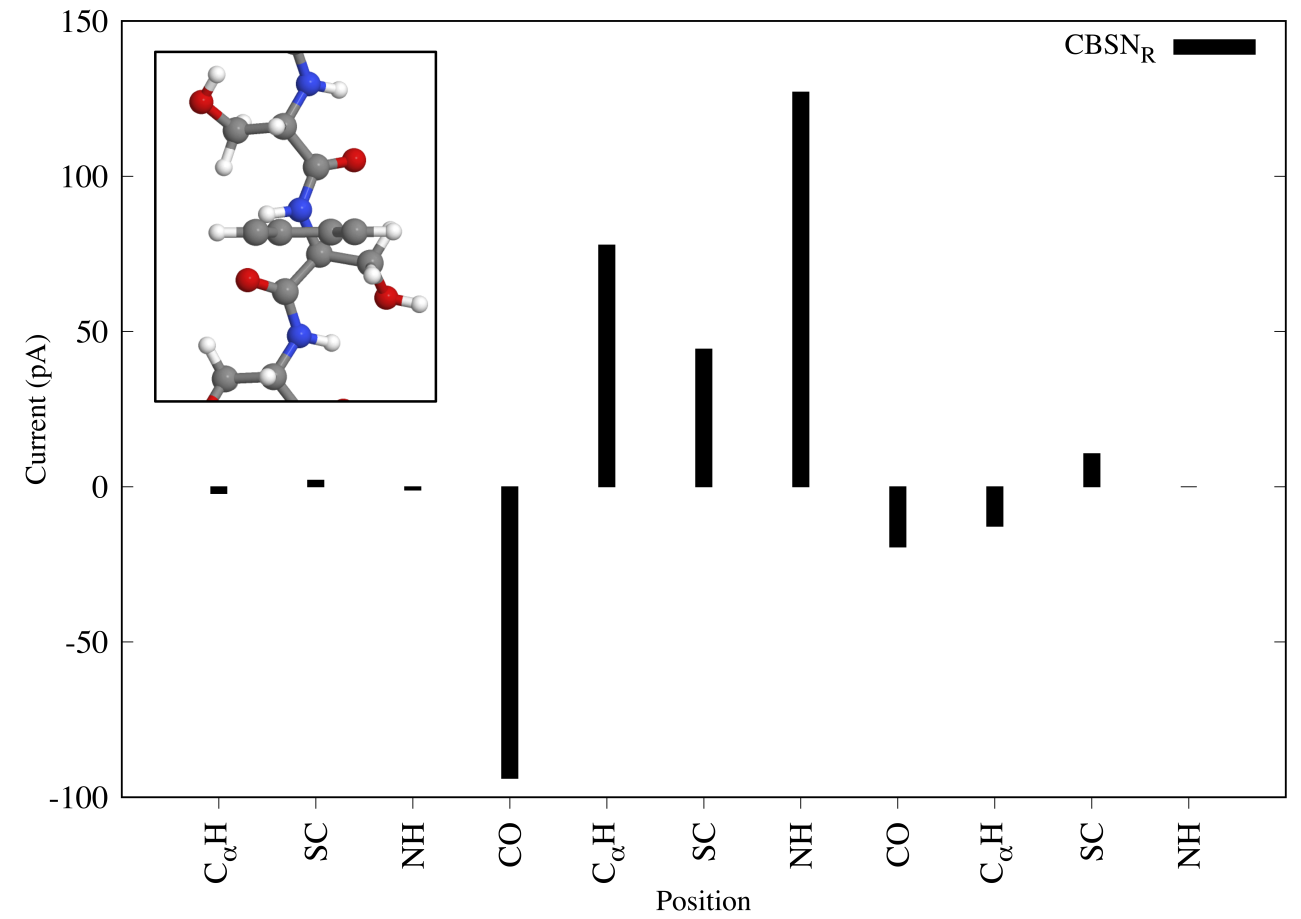

Figure S8: Bond currents injected from the left lead into the peptide for the $C B S N_{R}$ configuration of Ser homopeptide. In the boxes is reported the left lead side view of the configuration analyzed. 


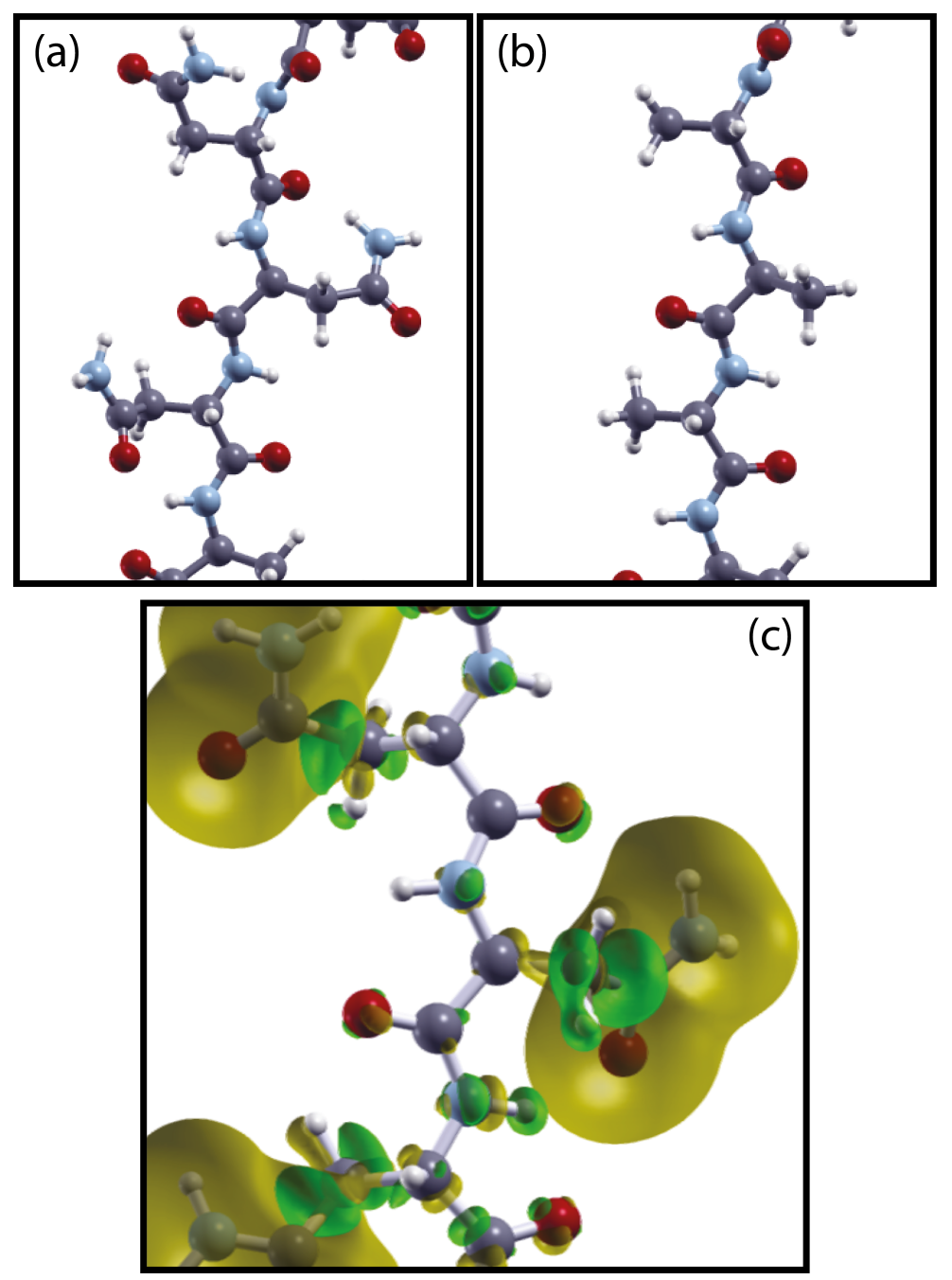

Figure S9: Analysis of the "polar" effect: differential electron density for a test case of the Asn homopeptide. We have considered the configuration corresponding to the peak at $\mathrm{CBSN}_{L}$. In (a) we show the Asn homopeptide. From this, we have removed the side chain polar ends that have been replaced with $\mathrm{H}$ atoms thus obtaining a new apolar configuration with the same atomic positions (except for the ones that have been replaced) (b). For both the Asn (polar) and the corresponding apolar configurations we have calculated the electron density maps of the $\mathrm{CBSN}_{L}$ configurations in the gap: their difference is reported in (c). We can see that the map of the electron density difference in the central backbone region of the peptide is very small, definitely negligible. This confirms that, as discussed in the main text, the polar side chains do not affect the tunneling current signal from the peptide backbone. 
a)

c)

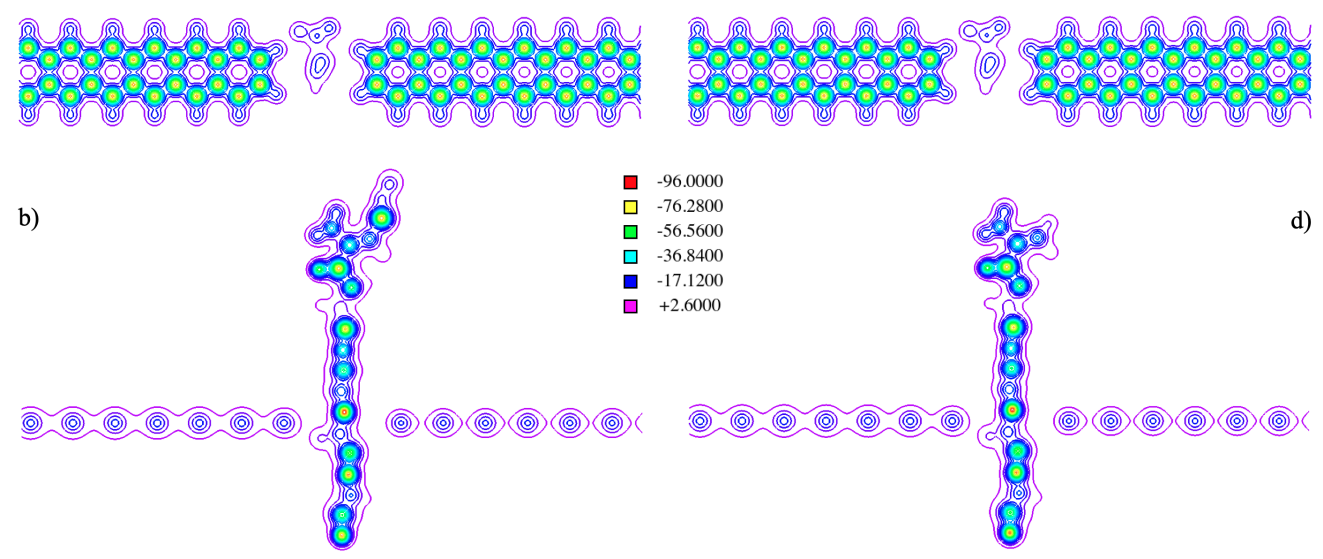

Figure S10: Analysis of the "polar" effect: electrostatic potential for a test case of the Asn homopeptide. Here we have still considered the polar configuration corresponding to the peak at $\mathrm{CBSN}_{L}$ (Fig. 9(a)) and the corresponding apolar one (Fig. 9(b)). Then we have calculated the total electrostatic potential energy maps for the polar, (a) top view and (b) side view, and the corresponding apolar configurations, (c) top view and (d) side view. We can see that the polar side chain does not affect the potential energy barrier. 